\title{
Distrofia muscular de Duchenne: reportes de
}

caso

\section{Duchenne Muscular Dystrophy: Case Reports}

Fecha de recepción: 15 Diciembre 2016 | Fecha de aprobación: 25 Mayo 2017

\author{
Víctor Hugo Guapi Nauñay \\ Hospital Provincial General Luis G. Dávila, Ecuador \\ Jorge Renán García Orbeb \\ Hospital Provincial General Luis G. Dávila, Ecuador
}

a Autor de correspondencia. Correo electrónico: victor_hg7@hotmail.com

b Autor de correspondencia. Correo electrónico: jrenan83@yahoo.es

Cómo citar: Guapi Nauñay VH, García Orbe JR. Distrofia muscular de Duchenne: reportes de caso. Univ Med. 2017;58(4):1-6. doi: http://dx.doi.org/1 0.11144/Javeriana.umed58-4.duch

\begin{abstract}
RESUMEN
La distrofia muscular de Duchenne es una enfermedad muscular grave ligada al cromosoma $\mathrm{X}$ que afecta el gen que codifica la distrofina, proteína fundamental para el mantenimiento de la fibra muscular. Se caracteriza por debilidad muscular de inicio en la infancia que sigue un curso progresivo. Sin intervención alguna, los pacientes pierden la marcha antes de la adolescencia y el fallecimiento ocurre en la segunda década de la vida por complicaciones respiratorias o problemas cardiacos. Actualmente no existe tratamiento curativo, pero la terapia con corticoides y el manejo multidisciplinario y ortopédico modifican la historia natural de esta miopatía. En este artículo se presentan dos casos clínicos de niños que presentaron dificultad para realizar actividades físicas vigorosas. Se les diagnosticó distrofia muscular de Duchenne confirmada por creatinafosfocinasa y electromiografía, con mejoría del cuadro clínico, gracias al tratamiento instaurado.

Palabras clave

distrofina; patrón de herencia; electromiografía; prednisona.
\end{abstract}

\begin{abstract}
The Duchenne muscular dystrophy is a bound serious muscular illness to the X-linked chromosome that affects to the gene that codes the dystrophin, protein important for the maintenance of the muscle fiber. It is characterized by muscle weakness of beginning in the childhood that follows a progressive course. Without intervention some, the patients lose the march before the younger and the death happens in the second decade of life for breathing complications or heart problems. At the moment it doesn't exist healing treatment, but the therapy with corticosteroids and the handling several disciplines and orthopedic they modify the natural history of this muscle disorders. In this article, we present two clinical cases of children who had difficulty prefoming vigorous physical actives. Diagnosis of Duchenne muscular dystrophy confirmed by creatin phosphokinase and electromyography with improvement of the clinical picture thanks to the treatment provided.

Keywords

dystrophin; inheritance patterns; electromyography; prednisone.
\end{abstract}




\section{Introducción}

La distrofia muscular de Duchenne (DMD) se caracteriza por una degeneración muscular progresiva que inicia en la infancia y confina al paciente a una silla de ruedas antes de los 12 años de edad, asociada a una afección de la musculatura cardiorrespiratoria $[1,2,3,4]$. Es una de las enfermedades monogénicas graves más frecuente; además, 1 de cada 3600-6000 se presenta en varones y es extremadamente rara en mujeres $[5,6]$.

Esta miopatía se transmite con un patrón de herencia recesivo ligado al cromosoma $\mathrm{X}$ y es el resultado de alteraciones en el gen de la distrofina, localizado en Xp21. El gen de la distrofina es el gen humano de mayor tamaño y posee 79 exones repartidos en $2300 \mathrm{~kb}$, codifica un mRNA de $14 \mathrm{~kb}$ y traduce una proteína de 3685 aminoácidos [2, 7].

Existe un extraordinario incremento en la actividad de la creatina-fosfocinasa (CPK) en el suero de pacientes con distrofia muscular [8, 9]. Existen otras enzimas que pueden aumentar su concentración en la sangre, como deshidrogenasa, transaminasa glutámico oxalacética (TGO) y transaminasa glutámico pirúvica (TGP), que están elevadas sin causa explicable $[5,10]$.

No existe tratamiento específico para la DMD, pero reportes de utilización de prednisona a dosis de 0,75 a $1,5 \mathrm{mg} / \mathrm{kg} /$ día han evidenciado que preserva la función muscular probablemente a través de la estabilización del sarcolema [11, 12].

\section{Métodos}

Se realizó un estudio observacional descriptivo de tipo reporte de casos con una exhaustiva anamnesis, examen físico y dismorfológico de los casos. Se arribó a una conclusión diagnóstica y a diagnósticos diferenciales con otras entidades.

En este artículo se presentan los casos de dos pacientes pediátricos en quienes se evidenciaron manifestaciones de miopatía dada por la debilidad muscular axial.

\section{Caso clínico 1}

El primer caso corresponde a un niño de ocho años de edad, nacido por parto eutócico a las 38 semanas de gestación, con adecuado peso y alta a los dos días. Acudió a nuestra consulta por remisión del departamento de rehabilitación por retraso del desarrollo, con dificultad para subir escaleras, correr, saltar y participar en actividades físicas vigorosas. Sus padres y su hermana están todos sanos, excepto su hermano, que está en iguales condiciones clínicas.

En el examen físico se observó que subía a la camilla de reconocimiento con dificultad; además, se puede objetivar debilidad axial al intentar incorporarse del decúbito, al igual que debilidad en la cintura pélvica, que obliga a levantarse del suelo con apoyo de los brazos sobre las piernas, maniobra de Gowers, marcha de pato, leve retracción aquilea o aumento del volumen de los gemelos, que a la palpación tienen una consistencia gomosa (seudohipertrofia) (figura $1)$.

Figura 1.Seudohipertrofia de los músculos gemelos, debido a la sustitución del tejido muscular por tejido conjuntivo y adiposo en el paciente de ocho años de edad, quien tiene DMD. Fotografía obtenida y publicada con el consentimiento informado de los padres

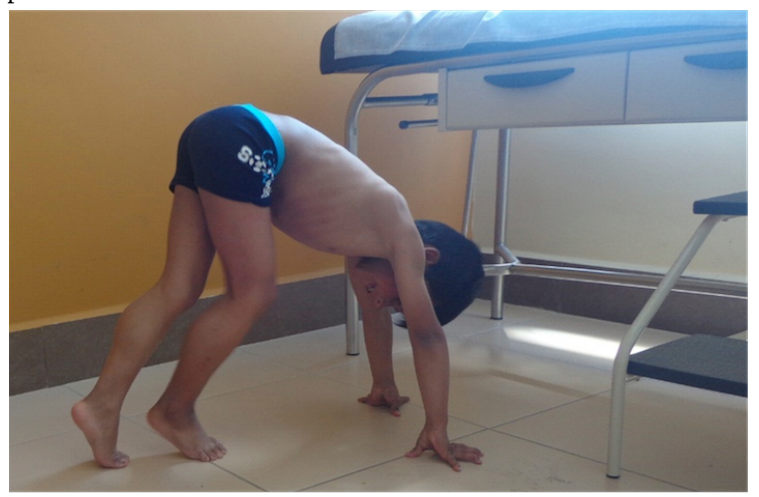

Antecedentes familiares: madre y padre de 25 y 29 años de edad, respectivamente, sanos, sin malformaciones. Parto intrahospitalario a término. No hay consanguinidad entre los padres.

Se realizó una interconsulta al servicio de cardiología, que reportó en el examen físico ausencia de cardiopatía. Los resultados de 
laboratorio fueron: TGO de 379 U/L; TGP de 293U/L; CPK de 9560,4 U/L, y electromiografía positiva para miopatía. Por la clínica y los exámenes complementarios se decidió iniciar (01/03/2016) de inmediato tratamiento con prednisona de $10 \mathrm{mg}$ vía oral cada $6 \mathrm{~h} \mathrm{(2}$ $\mathrm{mg} / \mathrm{kg} /$ día $\times 20 \mathrm{~kg}$ ) durante cuatro semanas. Posteriormente (01/04/2016) se reguló a la mitad de la dosis día $(20 \mathrm{mg})$, repartida en dos tomas por cuatro semanas. Finalmente (02/05/2016), una dosis de $5 \mathrm{mg}$ vía oral cada $12 \mathrm{~h}$. Después de iniciar el último esquema de corticoide se valoró la TGO, cuyo resultado fue de $146 \mathrm{U} / \mathrm{L}$, y la TGP, que presentó un valor de $286 \mathrm{U} / \mathrm{L}$ con marcada disminución en relación con los valores previos al tratamiento.

\section{Caso clínico 2}

El caso 2 corresponde a un niño de siete años de edad, nacido por parto eutócico a las 39 semanas de gestación, con adecuado peso y con alta a los tres días. La madre del paciente refiere retraso en el desarrollo psicomotor, caracterizado por tardanza en el sostén cefálico, para gatear, para ponerse de pie y en la deambulación misma.

En el examen físico se observó la maniobra de Gowers positiva, marcha de pato, retracción aquilea o aumento del volumen de los gemelos que a la palpación tienen una consistencia gomosa (seudohipertrofia); datos similares a los de su hermano (figura 2).
Figura 2.Paciente de siete años de edad quien tiene DMD. Demuestra la maniobra de Gowers. Fotografía obtenida y publicada con el consentimiento informado de los padres

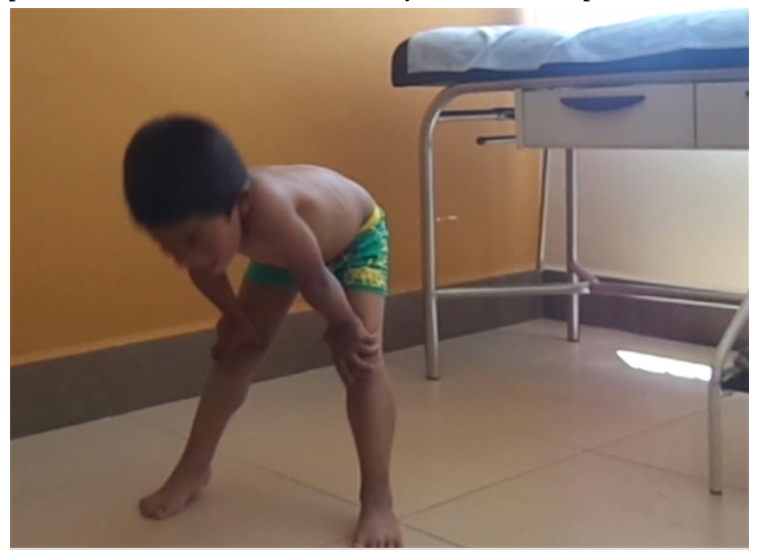

En este caso también se realizó interconsulta al servicio de cardiología, que reportó a la auscultación la no presencia de soplos. Los exámenes paraclínicos reportaron una TGO de $547 \mathrm{U} / \mathrm{L}$ y una TGP de $309 \mathrm{U} / \mathrm{L}$. Entre tanto, la CPK reportó un valor de 8450,3 U/L y la electromiografía fue positiva. Por la clínica y los exámenes complementarios, se decidió iniciar (01/03/2016) tratamiento con prednisona de $4 \mathrm{mg}$ vía oral cada $6 \mathrm{~h}(1 \mathrm{mg} / \mathrm{kg} / \mathrm{día} \times$ $16 \mathrm{~kg}$ ) durante cuatro semanas. Posteriormente (01/04/2016), se reguló a la mitad de la dosis día (8 mg) repartida en dos tomas. Después de iniciar el segundo esquema de corticoide se valoró la TGO, cuyo resultado fue de $125 \mathrm{U} / \mathrm{L}$, y la TGP, que marcó un valor de $286 \mathrm{U} / \mathrm{L}$, con una marcada disminución en relación con valores previos al tratamiento.

\section{Discusión}

La DMD es una enfermedad letal recesiva ligada al cromosoma $\mathrm{X}$; por ende, el riesgo de recurrencia en una mujer portadora de la enfermedad en cada gestación es en el $50 \%$ de hijos enfermos ; en el $50 \%$ de hijos sanos; en el $50 \%$ de hijas portadoras y en el $50 \%$ de hijas no portadoras. El llegar a un diagnóstico en determinada familia crea la necesidad de identificar a mujeres portadoras con la finalidad 
de establecer el adecuado asesoramiento genético $[2,3,13]$.

La presencia de un hijo afectado (IV-7) en la cuarta generación del familiograma (figura 3) identifica a la mujer III-7 como heterocigótica obligada con la probabilidad de otro varón afectado (IV-8) [2, 5, 13]. Es decir, los varones afectados (IV-7/IV-8) en la familia se relacionan unos con otros por medio de una mujer heterocigota (III-7) $[2,5]$. De la unión entre II-4 y II-5 se deduce que el individuo es afectado (II-4); por ende, la única hija (III-7) es portadora de la mutación, y como los hijos reciben el cromosoma Y de su padre, el hombre enfermo no tiene descendencia enferma, aunque transmite la mutación a sus nietos (IV-7/IV-8) por medio de sus hijas portadoras (III-7) [2, 14].

Figura 3. Genealogía de la familia con distrofia muscular de Duchenne

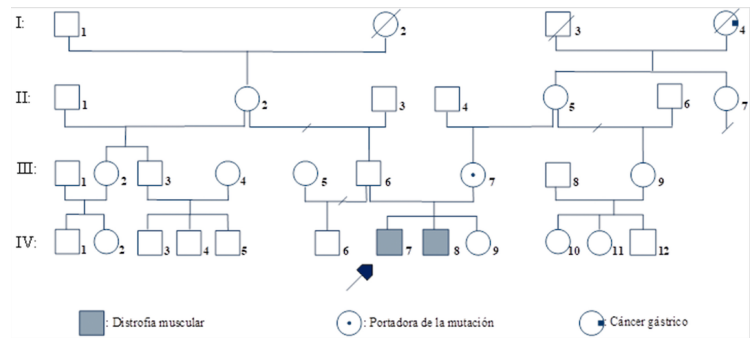

El fenotipo clínico y los resultados paraclínicos son homogéneos en la DMD, como lo expresado en los casos estudiados en este reporte; por tanto, el asesor genético debe conocer y emplear las diversas pruebas complementarias disponibles para establecer un diagnóstico preciso y brindar una oportuna atención médica no solo al paciente, sino a la familia [6, 14], puesto que los pacientes estudiados cursaron por consultas de diferentes profesionales de la salud (pediatría, endocrinología, rehabilitación, etc.), sin que profesional alguno definiera su verdadero diagnóstico.

La madre asegura que sus hijos presentaron retraso en la consecución de los objetivos del desarrollo, como gatear y caminar de forma independiente. Entre tanto, el rendimiento académico en el primero ha sido más tormentoso en relación con el segundo, expresado con alteraciones moderadas de lectura. Aunque la
DMD es una enfermedad muscular, puede asociar problemas cognitivos por la expresión a nivel cerebral de isoformas de la distrofina $[1,5]$. El coeficiente intelectual de los pacientes tiene una desviación estándar por debajo de la media y se puede encontrar deficiencia intelectual en 20-34 $\%$ de los casos $[2,8,15]$. Estos datos muestran la necesidad de una intervención temprana, ya que un inicio precoz de ayuda limitará problemas de aprendizaje y esto beneficiará su calidad de vida.

El diagnóstico diferencial de las entidades clínicas que presentan un signo de Gowers típico también se puede hallar en la dermatomiositis juvenil, la distrofia fascioescapulohumeral (enfermedad de Landouzy-Dejerine), la distrofia muscular de Becker y la distrofia muscular miotónica $[1,2,3,4,5]$.

En nuestros pacientes se podía descartar la dermatomiositis juvenil, por la ausencia de exantema, el dolor muscular o articular y los signos generales, como fiebre o pérdida de peso; todos muy frecuentes, aislados o en conjunción en este diagnóstico. La distrofia fascioescapulohumeral se caracteriza por debilidad precoz e intensa en los músculos faciales y de la cintura escapular y comienzo en general en la adolescencia [2, 3]. La distrofia muscular miotónica de Steinert suele iniciar en la adolescencia o la adultez, con afectación distal, facies típica y CPK normal o levemente aumentada. Por último, la distrofia muscular de Becker es fundamentalmente la misma enfermedad que la de Duchenne, con el defecto genético ligado al $\mathrm{X}$ y en el mismo locus, pero es menos frecuente (1 de 20.000 varones nacidos vivos) [3, 5]. Clínicamente, se manifiesta con una evolución más leve y prolongada e, histopatológicamente, se distingue por cantidades reducidas de distrofina $\mathrm{o}$, en ocasiones, una proteína cualitativamente anormal.

Aunque los tratamientos específicos para DMD aún no han llegado a la clínica, la historia natural de la enfermedad puede cambiar por la focalización de las intervenciones para las manifestaciones conocidas y las complicaciones. Los corticoides, así como las intervenciones ortopédicas, respiratorias, 
cardiacas y de rehabilitación han llevado a mejoras en la función, la calidad de vida, la salud y la longevidad, y los niños que son diagnosticados hoy en día tendrán la posibilidad de que su esperanza de vida llegue hasta la tercera década.

\section{Conclusiones}

Es necesario conocer y entender los aspectos relevantes de esta enfermedad, a fin de que sea diagnosticada precozmente; a su vez, se debe tener en consideración que existen otros tipos de distrofias musculares cuya información es necesaria para establecer el diagnóstico diferencial. Por supuesto, no debe faltar la elaboración del familiograma, herramienta que puede ayudar a orientar el diagnóstico o, incluso, a corroborarlo.

También es necesario un adecuado y detallado examen clínico que busque los signos y los síntomas propios de la enfermedad; realizar las pruebas complementarias pertinentes mencionadas en esta investigación; así como el estudio molecular de la alteración génica, todo en conjunto para definir el caso, y a la madre indicarle cuáles son las mujeres portadoras en la familia. En nuestro país, de momento, no se realiza el diagnóstico molecular, dato relevante para poder correlacionar genotipo-fenotipo.

Sin embargo, ante un paciente con sospecha clínica de DMD, el propósito y su familia entran en una evaluación clínica multidisciplinaria, incluido el asesoramiento genético para brindar una atención médica integral. La revisión de los casos presentados en este apartado brinda datos de interés sobre la evolución clínica de los pacientes, además del compromiso dirigido a médicos tratantes para que se actualizasen continuamente en los avances científicos referentes a esta enfermedad genética, y de esta manera ofrecer la alternativas de tratamiento que estén disponibles.

\section{Referencias}

1. Camacho A. Distrofia muscular de Duchenne. An Pediatric Contin. 2014;12(2):47-54.

2. Online Mendelian Inheritance in Man (OMIM) [internet]. 2015 [citado 2015 mar]. Disponible en: http://www.ncbi.nlm. nih.gov/Omim/

3. Turnpenny P, Ellard S. Emery's elements of medical genetics. 13a ed. Edimburgo: Elsevier Limited; 2007.

4. Biggar D. Duchenne muscular dystrophy. Peds in Review. 2015;27(3):83-8.

5. Lyons K. SMITH: patrones reconocibles de malformaciones humanas. 6a ed. Barcelona: Elsevier Saunders; 2008.

6. Mercier S, Toutain A, Toussaint A, Raynawd M, Barace C, Marcorelles P, et al. Genetic and clinical specifity of 26 symptomatic carriers for dystrophino pathies at pediatric age. Europ J H Gent. 2013;21(1):855-63.

7. Coral R, López L, Ruano L, Gómez B, Fernández F, Bahena E. Distrofias musculares en México: un enfoque clínico, bioquímico y molecular. Rev de Esp MedQuirur (México). 2014;15(3):152-60.

8. Kleinsteuber K, Avaria M. Enfermedades neuromusculares en pediatría. Rev Ped Elec. 2013;2(1);52-61.

9. Cammarata F, Camacho N, Alvarado J, Lacruz M. Distrofia muscular de Duchenne: presentación clínica. Rev Chil Pediatric. 2013;79(5):495-501.

10. Chaustre D, Chona W. Distrofia muscular de Duchenne: perspectivas de Rehabilitación. Rev Med. 2011;19(1):44-55.

11. Dietz H, Feero W, Guttmacher A. New therapeutic approaches to Mendelian disorders. N Engl J Med. 2012;363(9):852-63.

12. Bushby K, Finkel R, Birnkrant $\mathrm{D}$, Laura E, Clemens P, Cripe L, et al. Diagnóstico y tratamiento de la distrofia muscular de Duchenne. Lancet Neurol. 2014;9(1):77-93.

13. Lantigua A. Introducción a la genética médica. 2a ed. La Habana: Ciencias Médicas; 2011. 
Víctor Hugo Guapi Nauñay, Jorge Renán García Orbe.

14. Fonseca D, Arbeláez H, Silva C. Pérdida de heterocigocidad e identificación de portadores de distrofia muscular de Duchenne: un caso familiar con evento de recombinación. Rev Cienc Salud. 2012;10(1):83-90.

15. Ministerio de Salud Pública de Ecuador. Manual de prevención de riesgos de discapacidad en perineonatología. Quito: MSP; 2014. 